RU-97-05

hep-th/yymmxxx

May 17, 2018

\title{
Some Classical Solutions of Membrane Matrix Model Equations
}

\author{
Jens Hoppe \\ Institute for Theoretical Physics, \\ ETH Hönggerberg CH8093, Zürich, Switzerland \\ Department of Physics and Astronomy, Rutgers University, \\ Piscataway, New Jersey 08855-0849, USA
}

\begin{abstract}
Some exact solutions to the classical matrix model equations that arise in the context of $\mathrm{M}($ embrane) theory are given, and their topological nature is identified.
\end{abstract}


Let $X_{i}(t), i=1, \ldots, d$, be time-dependent elements of some (Lie-) algebra $\mathcal{A}$, satisfying (cp. ㄴ, 2, 匇, 团)

$$
\begin{gathered}
\ddot{X}_{i}=-\sum_{j=1}^{d}\left[\left[X_{i}, X_{j}\right], X_{j}\right] \\
\sum_{i=1}^{d}\left[X_{i}, \dot{X}_{i}\right]=0 .
\end{gathered}
$$

The Ansatz

$$
X_{i}(t)=x(t) r_{i j}(t) M_{j}
$$

(with $\left(r_{i j}\right)_{i j=1 \ldots d}=e^{\mathbf{A}(\mathbf{t})} \in S O(d)$ a time dependent rotation, $x(t)$ an overall pulsation, $\mathbf{A}(t)=\varphi(t) \mathbf{A}, \mathbf{A}^{2} \vec{M}=-\mu \vec{M}, x^{2}(t) \dot{\varphi}(t)=L=$ const.) reduces (1) to the equation(s)

$$
\sum_{j=1}^{d}\left[\left[M_{i}, M_{j}\right], M_{j}\right]=\lambda M_{i}
$$

while $x(t)$ is then given as a solution of $\ddot{x}+\lambda x^{3}-\frac{\mu L^{2}}{x^{2}}=0$, i.e.

$$
\frac{1}{2} \dot{x}^{2}+\frac{\lambda}{4} x^{4}+\frac{\mu L^{2}}{2} \frac{1}{x^{2}}=E
$$

for $L \neq 0$ (when $L=0,(2)$ automatically holds), (2) may e.g. be satisfied by choosing half (or more) of the components of $\vec{M}=\left(N_{1}, N_{2}, \ldots \underset{\sim}{N_{d}}, 0, \ldots, 0\right)$ to vanish, and $\mathbf{A}_{i j}$ to vanish whenever $M_{i}$ and $M_{j}$ don't, such as in

$$
X_{i}=x(t)(\cos \varphi(t) \vec{N}, \sin \varphi(t) \vec{N}, 0 \ldots 0) .
$$

Equation (4), which (for $\vec{M}^{2}=1$ ) may be considered as a discrete, or "quantized", analogue of minimally embedding a compact (2 dimensional) surface into a higher dimensional unit sphere (cp. [2], eq. (30)), has a very rich spectrum of solutionssome of which will now be given:

I) Let $\vec{N}$ be a basis of a representation of some Lie-algebra $\mathcal{G}$, satisfying

$$
\left[N_{a}, N_{b}\right]=i f_{a b c} N_{c}, \quad a, b, c=1, \ldots \underset{\sim}{\sim}, \quad f_{a b c} f_{c b a^{\prime}}=-\lambda \delta_{a a^{\prime}}
$$


I') Note that the Ansatz

$$
X_{a}(t)=X(t) \otimes N_{a}
$$

would reduce (1) to the (solvable) matrix model equations

$$
\ddot{X}+X^{3}=0
$$

with (2) being satisfied provided $[X(0), \dot{X}(0)]=0$.

II) Suppose $U, V \in \mathcal{A}$ satisfy

$$
V U=\omega U V
$$

with $\omega=e^{4 \pi i \Lambda}, \Lambda \in \mathbf{R}$;

$$
\vec{M}=\frac{1}{\sqrt{2}}\left(\frac{U+U^{-1}}{2}, \frac{U-U^{-1}}{2 i}, \frac{V+V^{-1}}{2}, \frac{V-V^{-1}}{2 i}, 0 \ldots 0\right)
$$

will then satisfy (4) with

$$
\lambda=2 \sin ^{2}(2 \pi \Lambda)
$$

-as for the above choice of $\vec{M}$ one has

$$
\sum_{j}\left[\left[\cdot, M_{j}\right], M_{j}\right]=\frac{1}{2}\left(\left[[\cdot, U], U^{-1}\right]+\left[[\cdot, V], V^{-1}\right]\right),
$$

which is proportional to the "quantum torus Laplace operator"

$$
\begin{gathered}
\Delta_{\Lambda}:=\frac{-1}{16 \pi^{2} \Lambda^{2}}\left(\left[[\cdot, U], U^{-1}\right]+\left[[\cdot, V], V^{-1}\right]\right), \\
\Delta_{\Lambda}\left(U^{m_{1}} V^{m_{2}}\right)=-\frac{\sin ^{2}\left(2 \pi \Lambda m_{1}\right)+\sin ^{2}\left(2 \pi \Lambda m_{2}\right)}{4 \pi^{2} \Lambda^{2}}\left(U^{m_{1}} V^{m_{2}}\right),
\end{gathered}
$$

whose eigenvalues for the 4 different components of $\vec{M}$ are identical. For rational $\Lambda$, $\Lambda=\frac{M}{N}, U$ and $V$ may be taken to be finite-dimensional matrices. The $N \rightarrow \infty$ limit of the solutions to the corresponding matrix equations (1) then either leads to solutions of the continuous membrane equations (cp. [1], [2]), (or choosing $M=M(N)$ such that $\frac{M}{N} \rightarrow \Lambda_{\infty} \neq 0$, see e.g. p. 53 of [5]) to solutions of the "star- product membrane equations" [6]. 
In the former case, it is known that the analogue of (4) has solutions describing minimal embeddings into $S^{3}$ of 2-dimensional surfaces of arbitrary genus (cp. [2]). The continuous limit of (11) and (7) $)_{S O(3)}$, e.g.,

$$
\begin{gathered}
\vec{m}=\frac{1}{\sqrt{2}}\left(\cos \varphi_{1}, \sin \varphi_{1}, \cos \varphi_{2}, \sin \varphi_{2}, 0 \ldots 0\right), \\
\vec{m}=(\sin \theta \cos \varphi, \sin \theta \sin \varphi, \cos \theta, 0 \ldots 0),
\end{gathered}
$$

(note that just as $\vec{m}^{2}=1,(11)$ also implies $\vec{M}^{2}=\mathbf{1}$, and in the case of (7) irreducible representations could be normalized to have $\vec{M}^{2}=1$ ) satisfy

$$
\left\{\left\{m_{i}, m_{j}\right\} m_{j}\right\}=2 m_{i}
$$

where $\{$,$\} denotes the Poisson brackets for functions on T^{2}$, resp. $S^{2}$.

III) Let

$$
\begin{array}{cl}
M_{1}=\frac{1}{4}\left(S_{k l}+S_{-k-l}+S_{-k l}+S_{k-l}\right), & M_{2}=\frac{-i}{4}\left(S_{k l}-S_{-k-l}-S_{-k l}+S_{k-l}\right), \\
M_{3}=\frac{-i}{4}\left(S_{k l}-S_{-k-l}+S_{-k l}-S_{k-l}\right), & M_{4}=\frac{-1}{4}\left(S_{k l}+S_{-k-l}-S_{-k l}-S_{k-l}\right),
\end{array}
$$

$M_{i>4}=0$, where

$$
S_{\vec{m}}:=\omega^{\frac{1}{2} m_{1} m_{2}} U^{m_{1}} V^{m_{2}},
$$

$\vec{m}=\left(m_{1}, m_{2}\right) \in \mathbf{Z}^{2}$, implying

$$
\begin{gathered}
S_{\vec{m}} S_{\vec{n}}=\omega^{-\frac{1}{2}\left(m_{1} m_{2}-m_{2} m_{1}\right)} S_{\vec{m}+\vec{n}}, \\
S_{\vec{n}} S_{\vec{m}} S_{-\vec{n}}=\omega^{(\vec{m} \times \vec{n})} S_{\vec{m}} .
\end{gathered}
$$

Using

$$
\sum_{i=1}^{4}\left[\left[X, M_{j}\right], M_{j}\right]=\frac{1}{2}\left(\left[\left[X, S_{k l}\right], S_{-k-l}\right]+\left[\left[X, S_{-k l}\right], S_{k-l}\right]\right) .
$$

and

$$
\left[\left[S_{\vec{m}}, S_{\vec{n}}\right], S_{-\vec{n}}\right]=4 \sin ^{2}(2 \pi \Lambda(\vec{m} \times \vec{n})) S_{\vec{m}}
$$


one finds that (18) satisfies (4), with

$$
\lambda=2 \sin ^{2}(2 \pi \Lambda(2 k l))
$$

and $\vec{M}^{2}=S_{00} \equiv \mathbf{1}$. (Note that, as long as $\Lambda$ is left arbitrary, the $k l$ degree of freedom is somewhat redundant, and without loss of generality one could have restricted to $k=1=l$, as was done in II). Also note that the continuous $(\Lambda \rightarrow 0)$ limit of $(19)$ gives minimal embedding into $S^{3}$ of two-dimensional surface with self-intersections, described by

$$
\vec{m}=\left(\operatorname{cosk} \varphi_{1} \operatorname{cosl} \varphi_{2}, \operatorname{sink} \varphi_{1} \operatorname{cosl} \varphi_{2}, \cos k \varphi_{1} \operatorname{sinl} \varphi_{2}, \operatorname{sink} \varphi_{1} \operatorname{sinl} \varphi_{2}, 0, \ldots 0\right)
$$

\section{Acknowledgement}

I would like to thank the Physics Departments of ETH Zürich and Rutgers University for their hospitality.

\section{References}

[1] J. Hoppe; "Quantum Theory of a Relativistic Surface", in: "Constraint's Theory and Relativistic Dynamics" (eds. G. Longhi, L. Lusanna) Arcetri, Florence 1986, World Scientific.

[2] J. Hoppe, H. Nicolai; Phys. Lett. B 196(1987)451.

[3] B. de Wit, J. Hoppe, H. Nicolai; Nucl. Phys. B305 [FS23](1988)545.

[4] T. Banks, W. Fischler, S.H. Shenker, L. Susskind; "M Theory as a Matrix Model: a Conjecture", hep-th/9610043.

[5] J. Hoppe, "Lectures On Integrable Systems", Springer Lecture Notes on Physics, 1992.

[6] J. Hoppe, Phys. Lett. B250(1990)44. 